\title{
Absence of sex differences in mental rotation performance in autism spectrum disorder
}

\author{
Melanie S Rohde', Alexandra L Georgescu',2, Kai Vogeley', \\ Rolf Fimmers $^{3}$ and Christine M Falter-Wagner ${ }^{1,4}$
}

\begin{abstract}
Mental rotation is one of the most investigated cognitive functions showing consistent sex differences. The 'Extreme Male Brain' hypothesis attributes the cognitive profile of individuals with autism spectrum disorder to an extreme version of the male cognitive profile. Previous investigations focused almost exclusively on males with autism spectrum disorder with only limited implications for affected females. This study is the first testing a sample of I 2 female adults with high-functioning autism spectrum disorder compared to 14 males with autism spectrum disorder, 12 typically developing females and 14 typically developing males employing a computerised version of the mental rotation test. Reaction time and accuracy served as dependent variables. Their linear relationship with degree of rotation allows separation of rotational aspects of the task, indicated by slopes of the psychometric function, and non-rotational aspects, indicated by intercepts of the psychometric function. While the typical and expected sex difference for rotational task aspects was corroborated in typically developing individuals, no comparable sex difference was found in autism spectrum disorder individuals. Autism spectrum disorder and typically developing individuals did not differ in mental rotation performance. This finding does not support the extreme male brain hypothesis of autism.
\end{abstract}

\section{Keywords}

adults, autism spectrum disorders, extreme male brain, mental rotation, sex differences, visuo-spatial

\section{Introduction}

The extent to which males and females differ in terms of cognitive profile has been a matter of research for decades. One of the most investigated domains of cognitive differences between typically developed (TD) females and males with a consistent advantage for males is the ability to rotate objects in mind, a function termed mental rotation (MR; Aleman et al., 2004; Astur et al., 2004; Brosnan et al., 2010b; Falter et al., 2008b; Linn and Petersen, 1985; Shepard and Metzler 1971; Tapley and Bryden, 1977; Voyer et al., 1995).

Autism spectrum disorders (ASDs) are pervasive developmental disorders with an unbalanced sex ratio of approximately four times as many affected males than females (Werling and Geschwind, 2013). Individuals with ASD are characterised by impairments in social interaction and communication, as well as restricted interests and stereotyped behaviour (American Psychological Association (APA), 2013). The so-called extreme male brain hypothesis (EMB) of autism (Baron-Cohen, 2002; Baron-Cohen et al.,
2005) rests on sex differences which are putatively found between females and males. EMB suggests ranking females and males along an empathising domain in which females are thought to excel and along a systemising domain in which males are thought to excel (Baron-Cohen, 2002; Baron-Cohen et al., 2005). Empathising refers to the ability of understanding and feeling others' emotions and reacting adequately, while systemising refers to the ability

\footnotetext{
'University Hospital of Cologne, Germany

¿University College London, UK

${ }^{3}$ Institut für Medizinische Biometrie, Informatik und Epidemiologie (IMBIE), Germany

${ }^{4}$ University of Munich (LMU), Germany
}

Corresponding author:

Christine M Falter-Wagner, Department of Psychiatry, Institute of Medical Psychology, University of Munich (LMU), Goethestr, 3I/ I, 80336 Munich, Germany.

Email: christine.falter@cantab.net 
of understanding, analysing and predicting rule-governed systems (Baron-Cohen, 2002). EMB theory defines ASD as an extreme version of the so-called 'male brain' or cognitive system with minimised empathic but emphasised systemising abilities, predicting superior performance of individuals with ASD in cognitive tasks in which males are assumed to outperform females. Typically, such sex differences in performance are most reliably found in MR tasks (see debate in Falter et al., 2008a; Knickmeyer et al., 2008).

The original MR task version by Shepard and Metzler (1971) was further developed (Falter et al., 2006) to the current computerised version in which participants are presented with pairs of three-dimensional figures, which are either different (mirrored) or the same but viewed from different perspectives $\left(0^{\circ}-120^{\circ}\right.$ of rotation). The participants' task is to decide as quickly and accurately as possible whether the figures are the same or not. Therefore, the figures must be kept in working memory and mentally rotated until a matching perspective is found (same trials) or not (different trials). Reaction time (RT) and accuracy (ACC) as dependent variables are measured and described as a psychometric function of degree of rotation allowing extraction of slopes and intercepts of the psychometric function. Slopes of individual psychometric functions have been operationalised as rotational aspects of the task (i.e. the function of mentally rotating a figure), while intercepts have been operationalised as non-rotational aspects of the task (i.e. working memory, stimulus encoding, comparison and decision-making processes, response preparation and execution; Gill et al., 1998; Hooven et al., 2004). Shallower slopes and lower intercepts for RT and higher intercepts for ACC indicate better performance.

Sex differences in TD individuals have usually been found in slopes, that is, the rotational aspect of MR (Brosnan et al., 2010b; Falter et al., 2006; Kozaki and Yasukouchi, 2009; Zapf et al., 2015). This is in contrast to studies comparing individuals from the two diagnostic groups of ASD and TD individuals which show differences in intercepts, that is, non-rotational aspects of MR (Falter et al., 2008b; Pearson et al., 2014). Our own studies (Falter et al., 2006, 2008b) used exactly the same task version, thus offering a direct comparison of performance differences between TD females and males with those between individuals with ASD and TD individuals. EMB would predict superior performance of individuals with ASD over TD individuals in the same domain (slopes or intercepts) in which TD males outperform TD females. Consequently, as argued by Falter et al. (2008a), findings of group differences in different domains thus challenge EMB theory.

However, some other studies using different MR task versions revealed differences between TD females and males in intercepts instead of slopes (Brosnan et al., 2010a, 2010b; Hooven et al., 2004). Also, while several studies showed superior performance of individuals with ASD over TD individuals (Falter et al., 2008b; Hamilton et al., 2009; Soulières et al., 2011), other studies failed to find a difference in MR performance between individuals with ASD and TD individuals (Beacher et al., 2012; Conson et al., 2013; Silk et al., 2006). Overall, a meta-analysis showed only an insignificant advantage for individuals with ASD over TD individuals for intercepts and none for slopes (Muth et al., 2014). However, even though not reflected in MR performance, studies employing functional magnetic resonance imaging (fMRI) revealed decreased neural activation among individuals with ASD in regions which are associated with working memory and executive functions (Hooven et al., 2004; Silk et al., 2006). Since those functions are assumed to be expressed in intercepts (Hooven et al., 2004), these findings support the assumption that any observed differences between individuals with ASD and TD individuals in the MR task reside in intercepts. Importantly, samples of individuals with ASD in most of the studies mentioned above were exclusively male or included only very few females with ASD. It is unclear to what extent EMB theory would predict a performance difference between males and females with ASD. Following the logic of testosterone-mediated MR performance differences, it would be consistent to assume a sex difference within ASD that is found in the same dimension of MR (whether intercepts or slopes) as the sex difference found in a TD sample.

Thus, the aim of this study was to establish whether sex differences exist in MR performance in ASD and to clarify whether they mirror sex differences found in a TD control sample. Inclusion and scrutiny of females with ASD in cognitive tasks is urgently needed as recent studies emphasise that females with ASD might show different performance patterns in some cognitive domains (Lehnhardt et al., 2016).

Employing the same validated computerised MR version in this study as previously (Falter et al., 2006, 2008b; Zapf et al., 2015) allows comparison across studies and a wider generalisation of results. In addition, Intuitive Physics Test (IPT; Baron-Cohen et al., 2001a) indexing systemising abilities was performed as suggested in previous studies (Brosnan et al., 2010a). Since systemising abilities are the crucial domain where males are thought to excel females in EMB, IPT was tested for correlations with MR performance. The ratio of the right second to fourth digit (2D:4D) was measured as proxy of prenatal testosterone levels (Manning et al., 2001) to test for correlations with MR performance. In normal populations, men were shown to have lower 2D:4D ratios than females (Lutchmaya et al., 2004; Manning et al., 2004) and individuals with ASD tend to have even lower 2D:4D ratios than normal populations (Manning et al., 2001). EMB directly links higher prenatal testosterone exposure to higher systemising abilities as well as lower empathy and to a higher risk for developing ASD, but this suggestion 
has been discussed controversially (see Hönekopp, 2012, for a review). This study replicates $2 \mathrm{D}: 4 \mathrm{D}$ ratio measurement to reevaluate previous findings showing that MR performance and digit ratios are unrelated (Falter et al., 2006, $2008 \mathrm{~b}$ ). Also, with respect to the heterogeneity of ASD and the broad range of degrees of severity, only participants with ASD as per diagnostic label of ICD10 and an IQ of at least one standard deviation (SD) under the group mean as well as Autism Spectrum Quotient (AQ) scores over 26 were included. A broad age range was chosen to allow comparability to other studies such as Brosnan et al. (2010a). Longitudinal data are lacking, but several studies suggest that visuo-spatial performance or executive functions in ASD do not depend on age (Guy et al., 2016; Ozonoff et al., 2004; Sachse et al., 2013) Finally, several comorbidities are observed to be over-represented in ASD, among them depression (Magnuson and Constantino, 2011; see Masi et al., 2017 for a recent review). EMB does not specify comorbidities as a limitation to the theory, but in order to control for possible influences on performance, depression was assessed and patients with comorbidities other than depression were not included.

\section{Methods}

\section{Participants}

A total of 34 individuals with ASD and 26 TD individuals were recruited. Ethical approval was granted by the Ethics Committee at the Medical Faculty, University of Cologne, and written informed consent was obtained before any testing. The age inclusion criterion for ASD participants was 20-55 years, ASD and TD participants were matched pairwise and matching allowed a maximum discrepancy of 6 years. Three individuals with ASD had to be excluded due to random response behaviour with less than $50 \%$ correct answers in conditions without rotation (i.e. $0^{\circ}$ conditions) and five had to be excluded because they did not meet the age criterion required to achieve group matching. Hence, 26 participants with ASD (12 females, 14 males) and 26 TD participants (12 females, 14 males) were included in the final analysis. Average ASD female age was 41.3 years and average ASD male age was 44.2 years. Average TD female age was 38.1 years and average TD male age was 44.3 years (see Table 1). All ASD participants were diagnosed and recruited in the Autism Outpatient Clinic at the Department of Psychiatry of the University Hospital of Cologne in Germany. All participants were tested individually. The experiment was performed in a testing room at the Center for Psychiatry of the University Hospital of Cologne in Germany equipped with facilities to perform paper-pencil tasks as well as a laptop with a $19^{\prime}$ screen to conduct the MR task. All participants performed the Wechsler Intelligenztest für Erwachsene (WIE; Aster et al., 2006). ASD and TD groups, as well as males and females, were matched with respect to age, verbal IQ and performance IQ (all $p \mathrm{~s}>0.05$, largest $t=-0.617$ ). Furthermore, Becks Depression Inventory (BDI) and AQ were measured. BDI (Beck and Brown, 2013) is a self-reporting inventory and consists of 21 multiple-choice questions designed to measure degree of depression. AQ (Baron-Cohen et al., 2001b) is a self-reporting questionnaire which consists of 50 questions revealing traits that are usually associated with autism. ASD and TD groups differed in BDI $(t(49)=4.080$, $p<0.001$ ) but only trends and no correlation with ACC or RT occurred (smallest $p=0.054$ ). As expected, AQ scores between ASD and TD groups differed significantly $(t$ $(49)=16.247, p<0.001)$. IPT (Baron-Cohen et al., 2001a) tests a participant's systemising abilities with 20 multiplechoice questions which should be answered in $10 \mathrm{~min}$. The more correct answers participants give, the better their intuitive physical understanding and the stronger their systemising tendencies. In addition, the length of the participant's right second and fourth digit from the basal crease to the tip of the digit was measured (2D:4D) with a ruler directly from the participant's hand after the experiment as proxy of prenatal testosterone exposure because higher levels of testosterone during ontogeny might be associated with higher risk for developing ASD (Manning et al., 2001) yet unrelated to MR performance (Falter et al., 2006, 2008b). Four individuals with ASD and three TD individuals refused measurement, so a total number of 22 individuals with ASD and 23 TD individuals entered this part of the analysis.

\section{Stimuli and design}

Participants conducted a computerised version of the Shepard and Metzler (1971) MR task (for specificities, see Falter et al., 2006). They were presented with pairs of three-dimensional figures and had to judge as fast and accurately as possible whether the two objects were the same or different (i.e. mirrored). The figures were viewed from a range of rotational angles: $0^{\circ}, 20^{\circ}, 40^{\circ}, 60^{\circ}, 80^{\circ}$, $100^{\circ}$ to $120^{\circ}$. The software Presentation (2014, version 17.0) was used for stimulus presentation and recording RT and number of correct answers for each participant which served as RT and ACC data for the final analysis. The experiment was conducted on a laptop with a $19^{\prime}$ screen. Before the experiment started, participants were given instructions and performed a test run of a minimum of 10 random trials taken from the original experiment until the experimenter was satisfied that the instructions were understood. After that, the experiment proper was started. The stimuli were light-blue three-dimensional figures presented on a black background. Their size was approximately $4^{\circ}$ of visual angle in width and height and each figure was constructed of 10 cubes (see Shepard and Metzler, 1971 for construction rules). In each trial, two stimuli were shown simultaneously on the right and left 
Table I. Demographic data for female and male ASD and TD participants.

\begin{tabular}{|c|c|c|c|c|c|c|}
\hline & & & Minimum & Maximum & Mean & SD \\
\hline \multirow[t]{14}{*}{ ASD } & Females $(N=12)$ & Age (years) & 20.17 & 48.83 & 41.33 & 8.71 \\
\hline & & VIQ & 81 & 130 & 104.33 & 15.20 \\
\hline & & PIQ & 67 & 126 & 101.42 & 17.93 \\
\hline & & BDI & 2 & 28 & 11.83 & 7.15 \\
\hline & & AQ & 27 & 48 & 41.75 & 5.50 \\
\hline & & IPT & 6 & 14 & 9.25 & 2.34 \\
\hline & & 2D:4D & 0.93 & 1.04 & 1.00 & 0.04 \\
\hline & Males $(N=14)$ & Age (years) & 29.08 & 51.58 & 44.23 & 6.42 \\
\hline & & VIQ & 88 & 135 & II 5.43 & $|4.2|$ \\
\hline & & PIQ & 80 & $14 \mid$ & 112.36 & 15.25 \\
\hline & & BDI & I & 27 & 11.43 & 8.78 \\
\hline & & $\mathrm{AQ}$ & 34 & 47 & 40.86 & 3.46 \\
\hline & & IPT & 4 & 15 & 7.64 & 2.84 \\
\hline & & 2D:4D & 0.95 & 1.04 & 1.00 & 0.03 \\
\hline \multirow[t]{14}{*}{ TD } & Females $(N=12)$ & Age (years) & 22.33 & 46.67 & 38.06 & 8.19 \\
\hline & & VIQ & 90 & 129 & 112.58 & 12.06 \\
\hline & & PIQ & 86 & 134 & 106.67 & 13.96 \\
\hline & & BDI & 0 & 14 & 6.33 & 5.02 \\
\hline & & $\mathrm{AQ}$ & 6 & 29 & 14.64 & 7.06 \\
\hline & & IPT & 6 & 14 & 9.50 & 2.35 \\
\hline & & 2D:4D & 0.97 & 1.10 & 1.02 & 0.04 \\
\hline & Males $(N=14)$ & Age (years) & 25.50 & 57.67 & 44.34 & 8.13 \\
\hline & & VIQ & 94 & 137 & 112.86 & 13.79 \\
\hline & & PIQ & 73 & 132 & 110.50 & 17.39 \\
\hline & & BDI & 0 & 9 & 2.31 & 2.87 \\
\hline & & $\mathrm{AQ}$ & 12 & 28 & 18.29 & 5.24 \\
\hline & & IPT & 6 & 16 & 10.54 & 2.57 \\
\hline & & 2D:4D & 0.96 & 1.07 & 1.01 & 0.03 \\
\hline
\end{tabular}

ASD: autism spectrum disorder; TD: typically developing; VIQ: verbal IQ; PIQ: performance IQ; BDI: Becks Depression Inventory; AQ: Autism Spectrum Quotient; IPT:Intuitive Physics Test; 2D:4D ratio: second to fourth digit ratio.

sides of the centre of the screen with a distance of approximately $10^{\circ}$ visual angle. Half of the trials were combinations of 'same' (rotated) figures and the other half were 'different' (mirrored and rotated) figures resulting in a total number of 448 trials presented in two blocks (Figure 1). Each combination of rotation angle and each pair of identical or mirrored objects were shown only once and in pseudo-randomised order. Participants were advised to record their decision by pressing one of two corresponding keyboard buttons with their index fingers. After key press, the figures disappeared. In case of a wrong answer, an acoustical feedback was given. The screen remained black for $500 \mathrm{~ms}$ before the next trial started.

After data collection was finished, slopes and intercepts of scores of ACC and RT were calculated as a measurement of MR abilities and statistically analysed to detect sex and group differences.

\section{Results}

Slopes and intercepts of accurate responses (ACC) and RTs were calculated and analysed separately using mixed analyses of variance (ANOVAs) with one betweenparticipants factor (GROUP: ASD vs TD) and one withinparticipants factor (CONDITION: same vs different). The expected main effect of CONDITION was confirmed for all dependent variables, ACC intercepts $(F(1,50)=43.992$, $\left.p=0.000, \eta^{2}=0.468\right)$ and RT intercepts $(F(1,50)=94.447$, $\left.p=0.000, \eta^{2}=0.654\right)$, as well as ACC slopes $(F(1$, $\left.50)=21.569, p=0.000, \eta^{2}=0.301\right)$ and RT slopes $(F(1$, $\left.50)=15.357, p=0.000, \eta^{2}=0.235\right)$, indicating same figures being easier to compare than different figures. No interaction (smallest $p=0.500$ ) and no main effect of GROUP were found (ACC intercepts: mean $\pm \mathrm{SD}=94.686 \pm 0.798$, $95 \%$ confidence interval $=[93.083 ; 96.289], p=0.132 ; \mathrm{RT}$ intercepts: $3084.807 \pm 181.015, \quad[2721.228 ; 3448.386]$, $p=0.583$; ACC slopes: $-0.161 \pm 0.013,[-0.187 ;-0.134]$, $p=0.794$; RT slopes: $21.579 \pm 2.477,[16.603 ; 26.554]$, $p=0.336$ ) which suggests that there was no performance difference between the TD and ASD groups in this study. In order to seek for sex differences within each diagnostic group, we performed separate mixed ANOVAs with one between-participants factor (SEX: female vs male) and one within-participants factor (CONDITION: same vs 


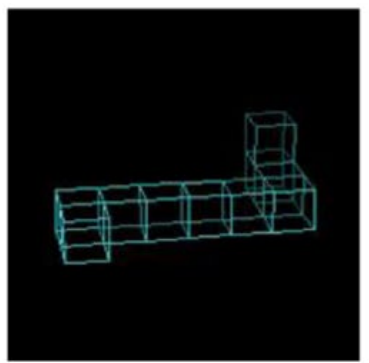

1

Examples for "same" trials (presented pairwise).

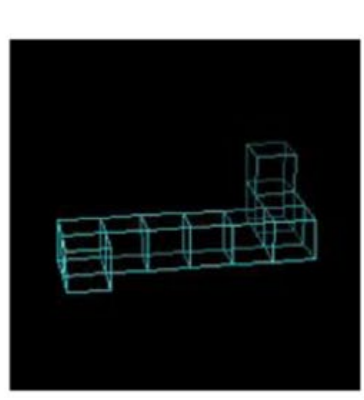

1

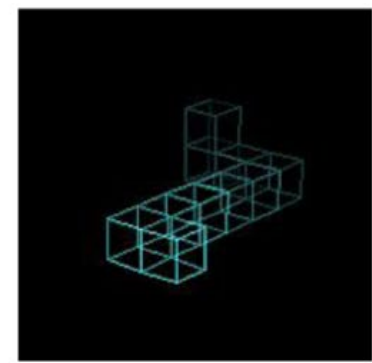

2

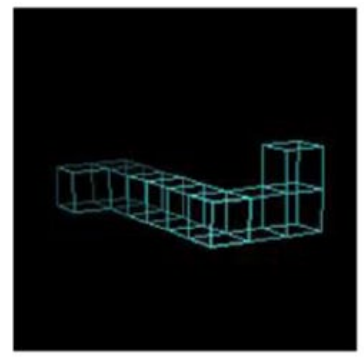

3

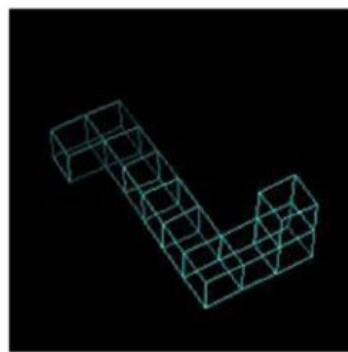

4

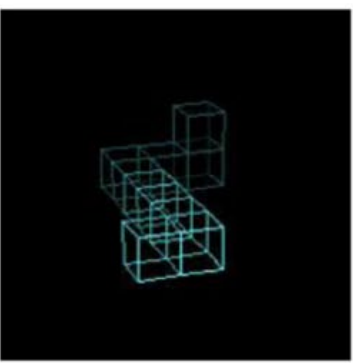

2

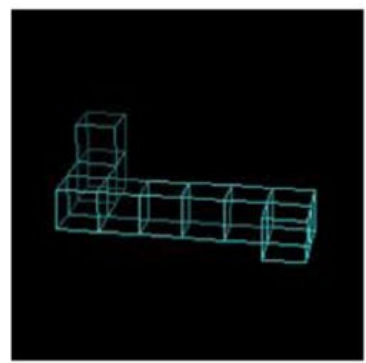

3

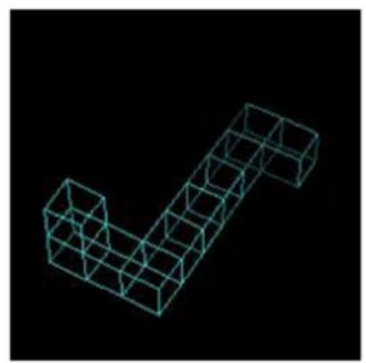

4

\section{Examples for "different" trials (presented pairwise).}

Figure I. Above, pictures I-4 show examples for identical objects ('same' trials) from different rotation angles $\left(0^{\circ}-120^{\circ}\right)$. In each trial, two of them were presented pairwise and participants were advised to signal their judgment by pressing the corresponding key. Below, pictures $\mathrm{I}-4$ show examples for mirrored objects from different rotation angles $\left(0^{\circ}-120^{\circ}\right)$ which were presented pairwise.

different) for both diagnostic groups separately. Besides the same effect of CONDITION as given above, there was a main effect of SEX in the TD group for RT slope $(F(1$, $\left.24)=5.196, p=0.032, \eta^{2}=0.178\right)$. Concerning intercepts, there were only trends for significance for RT in the TD group $\left(F(1,24)=3.126, p=0.090, \eta^{2}=0.115\right)$ and ACC in the ASD group $\left(F(1,24)=3.077, p=0.092, \eta^{2}=0.114\right)$. No interaction was found (smallest $p=0.057$ ) and no other main effects of SEX in either the TD or ASD group occurred (ACC intercepts: (mean ASD, TD \pm SD ASD, $\mathrm{TD})=(95.771,93.560 \pm 1.027,1.188), 95 \%$ confidence interval $=[93.650,91.107 ; 97.891,96.012], p=0.092$, 0.298; RT intercepts: $(3000.156,3154.542 \pm 283.003$, 221.813), [2416.066, 2696.743; 3584.246, 3612.341], $p=0.490,0.090$; ACC slopes: $(-0.156,-0.164 \pm 0.018$, $0.020),[-0.193,-0.205 ;-0.120,-0.123], p=0.365,0.820$; RT slopes: $(24.337,19.483 \pm 4.583,1.782), \quad[14.879$, $15.805 ; 33.795,23.160], p=0.332,0.032)$. With respect to 2D:4D ratios, there was only a trend of a group difference between ASD and TD groups $(t(43)=-1.805 ; p=0.078)$ and there were no SEX differences in 2D:4D ratios within both diagnostic groups (smallest $p=0.362$ ). Neither GROUP nor SEX differences were found for IPT (smallest $p=0.123)$. For the ASD group, correlations between IPT and ACC slopes for same trials $(r=-0.628 ; p=0.001)$, as well as ACC intercepts for different trials $(r=-0.750$; $p<0.001$ ), were significant (Bonferroni-Holm corrected alpha-values). No significant correlations occurred for the TD group (smallest $p=0.266$ ) (Figures 2 and 3; Table 2).

\section{Discussion}

Stimulated by recent evidence for differences in the cognitive profile between females and males with ASD (Lehnhardt et al., 2016), the aim of this study was to test whether typically found sex differences between TD females and males (Aleman et al., 2004; Astur et al., 2004; Brosnan et al., 2010b; Falter et al., 2008b; Linn and Petersen, 1985; Tapley and Bryden, 1977; Voyer et al., 1995) in a classical MR task (Shepard and Metzler, 1971) would also be reflected in performance differences between females and males with ASD. To our knowledge, this is the first study which directly compares MR performance between ASD females and males. Furthermore, we sought 


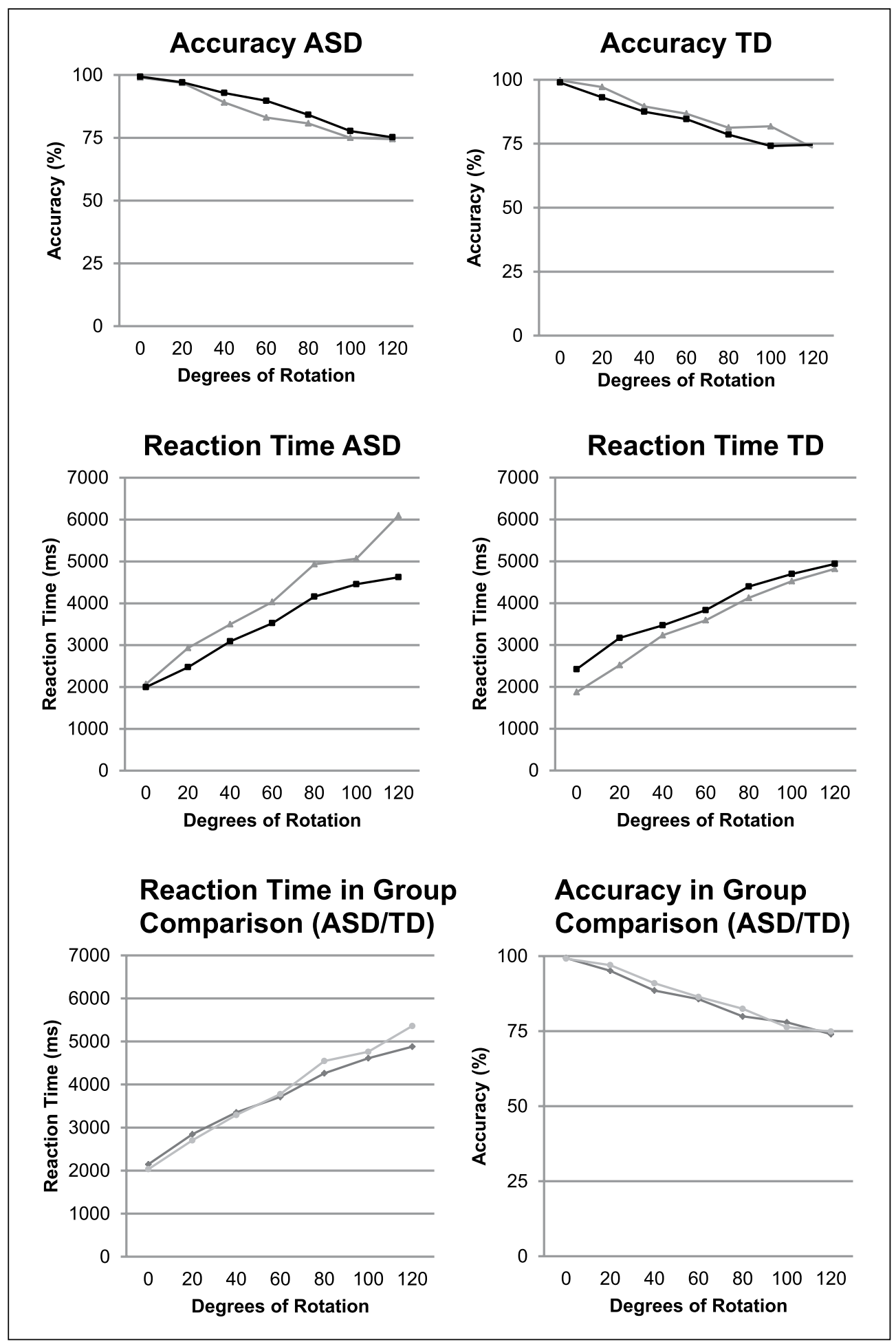

Figure 2. Comparison of MR reaction time (RT) and accuracy (ACC) scores between males (black squares) and females (grey triangles) for the ASD and TD groups separately (upper four panels) and comparison of RT and ACC scores between ASD and TD (bottom panels). Dark grey diamonds represent the TD group; light grey circles represent the ASD group.

to clarify in which aspects of the MR task TD and ASD females and males differ. This issue has been addressed before but discussed controversially (e.g. Falter et al., 2008b; Brosnan et al., 2010a, 2010b). In accordance with previous findings (e.g. Brosnan et al., 2010a, 2010b; Falter et al., 2006; Zapf et al., 2015), males in this study were 


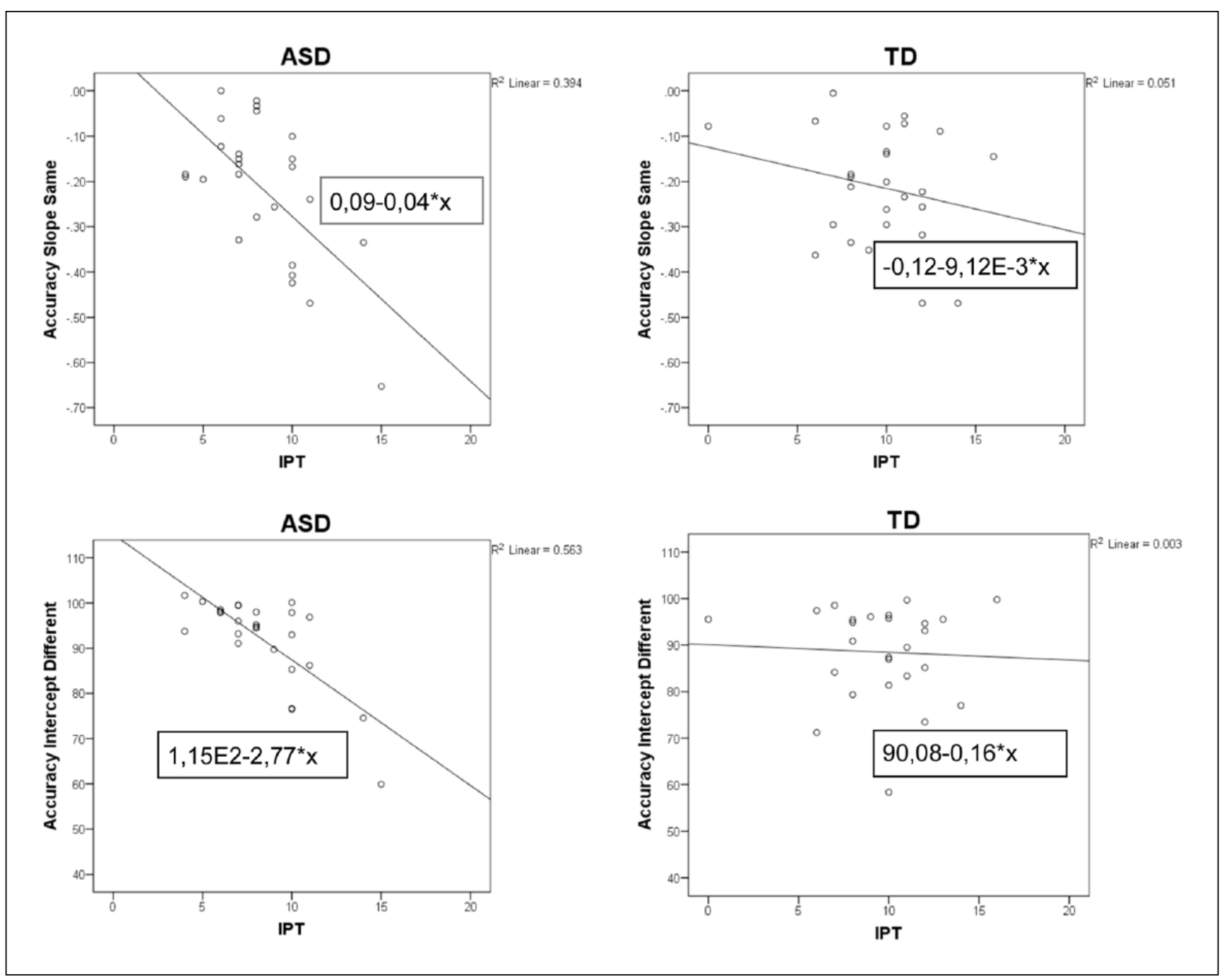

Figure 3. Comparison of correlations between IPT and accuracy slopes for same trials and accuracy intercepts for different trials for ASD and TD group.

found to outperform females. Although originally designed as a visuo-spatial task, MR performance requires both visuo-spatial skills per se (i.e. mentally rotating objects in space called rotational skills) represented by slopes as well as non-rotational aspects (such as figure comparison, decision-making, response preparation and working memory) represented by intercepts. Despite of a few reports of sex differences in non-rotational task aspects (Brosnan et al., 2010a; Hooven et al., 2004), the majority of studies to date found sex differences to reside only in rotational task aspects (Brosnan et al., 2010b; Falter et al., 2006, 2008b; Kozaki and Yasukouchi, 2009; Zapf et al., 2015). As corroborated by the current findings, there is now converging evidence for sex differences in TD to reside in the domain of rotational task aspects. This bears implications on EMB theory as argued by Falter et al. (2008a, 2008b; see discussion in Knickmeyer et al., 2008). If, as predicted by EMB theory, individuals with ASD show an extreme version of male cognitive skill patterns, then superior performance would be expected in the same domain in which typical sex differences are found.

However, a few studies previously showed differences in non-rotational aspects of the MR task (Brosnan et al., 2010a, 2010b; Hooven, et al., 2004). In these studies, it has been argued that testosterone facilitates MR abilities, which is reflected in male superiority over females in nonrotational task aspects. For example, Hooven et al. (2004) found in a sample of TD males that higher circulating testosterone was associated with lower error rates and faster RT, but only for non-rotational and not for rotational task aspects. Similarly, Brosnan et al. (2010a) tested TD females and males and found a relation between daytime, a proxy for circulating testosterone and non-rotational MR aspects. Note though that studies investigating testosterone levels must be interpreted with caution given various factors are known to influence testosterone levels in females and males such as daytime (see Valdez et al., 2008 for a summary), exercise (Hulmi et al., 2008), menstrual 


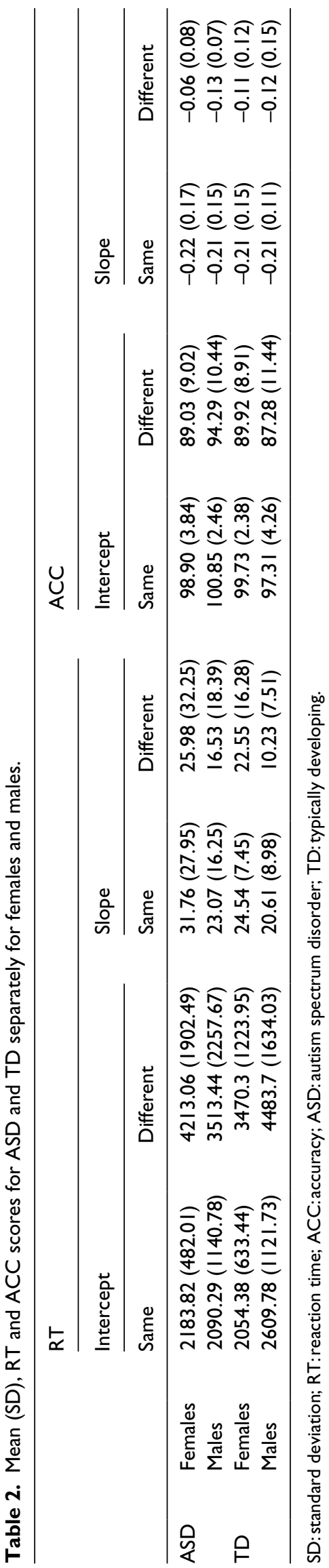

cycle (Celec et al., 2002) or natural fluctuations (Courvoisier et al., 2013). Also, intercepts themselves are potentially suspect to arousal levels as noted by Falter et al. (2006). As argued, videos showing either sexual or dentistry content used in the study by Hooven et al. (2004) to manipulate testosterone levels could have given rise to different arousal levels in high- compared to low-testosterone males leading to differences in intercepts that might not primarily be caused by testosterone.

Overall, the picture drawn by studies on testosterone is inconsistent (see Falter et al., 2006 for a summary) and lacks further research. Many studies found no association between testosterone and spatial abilities in general (e.g. McKeever et al., 1987) or, more specifically, between testosterone and MR (Alexander et al., 1998; Falter et al., 2008b; Halari et al., 2005). Although testosterone was not the focus of our study, we could neither show the reported differences in non-rotational task aspects nor did we find systemising to correlate with MR, although systemising has previously been shown to correlate with a proxy for circulating testosterone and better performance in nonrotational task aspects (Brosnan et al., 2010a). Instead, we replicated previous findings that fetal testosterone indexed by 2D:4D ratios (Manning et al., 2001) is not related to systemising (Voracek and Dressler, 2006) or MR performance (Falter et al., 2006, 2008b). Taken together, the lack of relationship between MR performance and both systemising and 2D:4D ratios in this study as well as an increasing number of studies corroborating the idea that typical sex differences observed in MR usually reside in rotational task aspects (Brosnan et al., 2010b; Falter et al., 2006; Kozaki and Yasukouchi, 2009; Zapf et al., 2015) add to the growing and converging body of evidence against cognitive profile predictions of EMB theory (see Falter et al., 2008a).

Contrary to previous research (Brosnan et al., 2010a; Zapf et al., 2015), we did not find the reported sex differences in IPT which might be due to less statistical power given a smaller number of participants in this study. Interestingly, Brosnan et al. (2010a), found systemising to correlate only with non-rotational aspects of MR, specifically accuracy intercepts for same and different responses, and they found sex differences in the same aspect of MR. In contrast, systemising in our study correlated with rotational and non-rotational parts of MR, specifically slopes of accuracy for same trials and intercepts of accuracy for different trials, and we also found sex differences in rotational components of MR. These current findings correspond to previous findings by Zapf et al. (2015) who also found a correlation between IPT and rotational aspects of MR. Consequently, systemising tends to be rather associated with rotational than non-rotational aspects of MR. Besides that, a closer view on the correlations described above and the correlations found by Zapf et al. (2015) shows a negative correlation between systemising and 
$\mathrm{MR}$, indicating that better systemising abilities go along with worse performance in MR, while with respect to EMB, one would expect a positive relationship between systemising and superior visuo-spatial performance.

Same and different comparisons yielded significantly different performance in line with the findings of the original Shepard and Metzler (1971) paradigm, indicating that 'different' trials are more difficult because MR of the figures needs to be completed to reach the conclusion that they do not match. For 'same' trials, rotation only needs to be performed up to the degree of match (for discussion, see Brosnan et al., 2010b; Pearson et al., 2014). Nevertheless, there was no interaction of 'same' versus 'different' trials with sex, showing that this distinction is irrelevant for the question of sex differences in MR performance.

Compared to the numerous studies on MR in TD individuals, studies on MR in ASD are rather rare. In a previous meta-analysis, it was found that individuals with ASD do not have a generalised deficit in visuo-spatial abilities. MR performance varied between studies and showed no overall meta-analytic effect between groups, neither for slopes nor for intercepts (Muth et al., 2014). There was only a weak population effect size for performance differences in MR between ASD and TD located in intercepts and no overall superior performance of individuals with ASD in RT slopes (Muth et al., 2014). We also sought to investigate possible sex differences in MR performance within a group of individuals with ASD and probed consistency of results with EMB theory. Given that female ASD performance in our study is located between TD female and male performance, there is no ceiling effect of MR which might hide sex differences among ASD. Contrary to EMB predictions, we could not verify superior performance of ASD compared to TD individuals. EMB does not explicitly predict cognitive sex differences between ASD females and males, but following its logic, MR performance of ASD females should be better than the performance of TD males and equivalent to the performance of ASD males. However, this pattern could not be underpinned by this study.

The current literature is showing an emerging evidence for a female ASD cognitive profile which is different from the cognitive profile that males with ASD show (Frazier et al., 2014; Lai et al., 2011; see Lehnhardt et al., 2016 for a recent summary). While ASD males show superior performance in visuo-spatial tasks, ASD females outperform their male counterparts in terms of processing speed (Bölte et al., 2011; Koyama et al., 2009; Lehnhardt et al., 2016) and executive functions (Lehnhardt et al., 2016) but they perform worse in terms of working memory (Nydén et al., 2000). Those functions are required in different aspects of MR but performance differences between ASD females and males in certain cognitive domains might be covered by an equal general performance in MR. For example, executive functions and working memory are both encoded in intercepts (Hooven et al., 2004) but while females excel in one domain, they might not in the other domain. Speculatively, overall performance in an MR task might therefore not differ. Although such a scenario might give a partial explanation for the results of this study, it would not fully explain why MR as the most robust test for sex differences in TD does not reveal sex differences in ASD. However, since there is no test comparable to MR in terms of sex differences in the TD population, it might be challenging but necessary to find a different approach to reevaluate visuo-spatial sex differences in ASD in future research.

In conclusion, this study uncovers a lack of sex difference in ASD in terms of visuo-spatial skills. Importantly, it contributes to the growing body of studies showing that individuals with ASD do not always display an exaggerated male cognitive profile and that females with ASD need particular characterisation and research attention in the future.

\section{Funding}

The author(s) received no financial support for the research, authorship and/or publication of this article.

\section{References}

Aleman A, Bronk E, Kessels RP, et al. (2004) A single administration of testosterone improves visuospatial ability in young women. Psychoneuroendocrinology 29(5): 612-617.

Alexander GM, Swerdloff RS, Wang C, et al. (1998) Androgenbehavior correlations in hypogonadal men and eugonadal men. II. Cognitive abilities. Hormones and Behavior 33(2): 85-94.

American Psychological Association (APA) (2013) Diagnostic and Statistical Manual of Mental Disorders. 5th ed. Arlington, VA: American Psychiatric Publishing.

Aster M, Neubauer A and Horn R (2006) Wechsler Intelligenztest für Erwachsene (WIE). Deutschsprachige Bearbeitung und Adaptation des WAIS-III von David Wechsler. Frankfurt: Harcourt Test Services.

Astur RS, Tropp J, Sava S, et al. (2004) Sex differences and correlations in a virtual Morris water task, a virtual radial arm maze, and mental rotation. Behavioural Brain Research 151(1-2): 103-115.

Baron-Cohen S (2002) The extreme male brain theory of autism. Trends in Cognitive Sciences 6(6): 248-254.

Baron-Cohen S, Knickmeyer RC and Belmonte MK (2005) Sex differences in the brain: implications for explaining autism. Science 310(5749): 819-823.

Baron-Cohen S, Wheelwright S, Scahill V, et al. (2001a) Are intuitive physics and intuitive psychology independent? A test with children with Asberger syndrome. Journal of Developmental and Learning Disorders 5: 47-78.

Baron-Cohen S, Wheelwright S, Skinner R, et al. (2001b) The autism-spectrum quotient (AQ): evidence from Asperger syndrome/high-functioning autism, males and females, scientists and mathematicians. Journal of Developmental and Learning Disorders 31(1): 5-17. 
Beacher FD, Radulescu E, Minati L, et al. (2012) Sex differences and autism: brain function during verbal fluency and mental rotation. PLoS ONE 7(6): e38355.

Beck AT and Brown GK (2013) Beck-Depressions-Inventar-FS (BDI-FS) Manual (Deutsche Bearbeitung von Sören Kliem and Elmar Brähler). Frankfurt am Main: Pearson Assessment.

Bölte S, Duketis E, Poustka F, et al. (2011) Sex differences in cognitive domains and their clinical correlates in higher-functioning autism spectrum disorders. Autism 15(4): 497-511.

Brosnan M, Daggar R and Collomosse J (2010a) The relationship between systemising and mental rotation and the implications for the extreme male brain theory of autism. Journal of Autism and Developmental Disorders 40(1): $1-7$.

Brosnan M, Walker I and Collomosse J (2010b) The effect of explicitly varying the proportion of 'same' and 'different' responses on sex differences in the Shepard and Metzler mental rotation task. European Journal of Cognitive Psychology 22: 172-189.

Celec P, Ostatnikova D, Putz Z, et al. (2002) The circalunar cycle of salivary testosterone and the visual-spatial performance. Bratislavske Lekarske Listy 103(2): 59-69.

Conson M, Mazzarella E, Frolli A, et al. (2013) Motor imagery in Asperger syndrome: testing action simulation by the hand laterality task. PLoS ONE 8(7): e70734.

Courvoisier DS, Renaud O, Geiser C, et al. (2013) Sex hormones and mental rotation: an intensive longitudinal investigation. Hormones and Behavior 63(2): 345-351.

Falter CM, Arroyo M and Davis GJ (2006) Testosterone: activation or organization of spatial cognition? Biological Psychology 73(2): 132-140.

Falter CM, Plaisted KC and Davis G (2008a) Male brains, androgen, and the cognitive profile in autism: convergent evidence from 2D:4D and congenital adrenal hyperplasia. $J$ Autism Dev Disord 38(5): 997-998.

Falter CM, Plaisted KC and Davis G (2008b) Visuo-spatial processing in autism - testing the predictions of extreme male brain theory. Journal of Autism and Developmental Disorders 38(3): 507-515.

Frazier TW, Georgiades S, Bishop SL, et al. (2014) Behavioral and cognitive characteristics of females and males with autism in the Simons Simplex Collection. Journal of the American Academy of Child and Adolescent Psychiatry 53(3): 329-340.e1-3.

Gill HS, O'Boyle MW and Hathaway J (1998) Cortical distribution of EEG activity for component processes during mental rotation. Cortex 34(5): 707-718.

Guy J, Mottron L, Berthiaume C, et al. (2016) A developmental perspective of global and local visual perception in autism spectrum disorder. Journal of Autism and Developmental Disorders. Epub ahead of print 1 July. DOI: 10.1007/ s10803-016-2834-1.

Halari R, Hines M, Kumari V, et al. (2005) Sex differences and individual differences in cognitive performance and their relationship to endogenous gonadal hormones and gonadotropins. Behavioral Neuroscience 119(1): 104-117.

Hamilton AF, Brindley R and Frith U (2009) Visual perspective taking impairment in children with autistic spectrum disorder. Cognition 113(1): 37-44.
Hönekopp J (2012) Digit ratio 2D:4D in relation to autism spectrum disorders, empathizing, and systemizing: a quantitative review. Autism Research 5(4): 221-230.

Hooven CK, Chabris CF, Ellison PT, et al. (2004) The relationship of male testosterone to components of mental rotation. Neuropsychologia 42(6): 782-790.

Hulmi JJ, Ahtiainen JP, Selänne H, et al. (2008) Androgen receptors and testosterone in men-effects of protein ingestion, resistance exercise and fiber type. Journal of Steroid Biochemistry and Molecular Biology 110(1-2): 130-137.

Knickmeyer RC, Baron-Cohen S, Auyeung B, et al. (2008) How to test the extreme male brain theory of autism in terms of foetal androgens? Journal of Autism and Developmental Disorders 38(5): 995-996; author reply 7-8.

Koyama T, Kamio Y, Inada N, et al. (2009) Sex differences in WISC-III profiles of children with high-functioning pervasive developmental disorders. Journal of Autism and Developmental Disorders 39(1): 135-141.

Kozaki T and Yasukouchi A (2009) Sex differences on components of mental rotation at different menstrual phases. International Journal of Neuroscience 119(1): 59-67.

Lai MC, Lombardo MV, Pasco G, et al. (2011) A behavioral comparison of male and female adults with high functioning autism spectrum conditions. PLOS ONE 6(6): e20835.

Lehnhardt FG, Falter CM, Gawronski A, et al. (2016) Sex-related cognitive profile in autism spectrum disorders diagnosed late in life: implications for the female autistic phenotype. Journal of Autism and Developmental Disorders 46(1): 139-154.

Linn MC and Petersen AC (1985) Emergence and characterization of sex differences in spatial ability: a meta-analysis. Child Development 56(6): 1479-1498.

Lutchmaya S, Baron-Cohen S, Raggatt P, et al. (2004) 2nd to 4th digit ratios, fetal testosterone and estradiol. Early Human Development 77(1-2): 23-28.

McKeever WF, Rich DA, Deyo RA, et al. (1987) Androgens and spatial ability: failure to find a relationship between testosterone and ability measures. Bulletin of the Psychonomic Society 25: 438-440.

Magnuson KM and Constantino JN (2011) Characterization of depression in children with autism spectrum disorders. Journal of Developmental and Behavioral Pediatrics 32: 332-340.

Manning JT, Baron-Cohen S, Wheelwright S, et al. (2001) The 2nd to 4 th digit ratio and autism. Developmental Medicine and Child Neurology 43(3): 160-164.

Manning JT, Stewart A, Bundred PE, et al. (2004) Sex and ethnic differences in 2 nd to 4 th digit ratio of children. Early Human Development 80: 161-168.

Masi A, DeMayo MM, Glozier N, et al. (2017) An overview of autism spectrum disorder, heterogeneity and treatment options. Neuroscience Bulletin 33: 183-193.

Muth A, Hönekopp J and Falter CM (2014) Visuo-spatial performance in autism: a meta-analysis. Journal of Autism and Developmental Disorders 44(12): 3245-3263.

Nydén A, Hjelmquist E and Gillberg C (2000) Autism spectrum and attention-deficit disorders in girls. Some neuropsychological aspects. European Child \& Adolescent Psychiatry 9(3): 180-185. 
Ozonoff S, Cook I, Coon H, et al. (2004) Performance on Cambridge Neuropsychological Test Automated Battery subtests sensitive to frontal lobe function in people with autistic disorder: evidence from the Collaborative Programs of Excellence in Autism network. Journal of Autism and Developmental Disorders 34(2): 139-150.

Pearson A, Marsh L, Hamilton A, et al. (2014) Spatial transformations of bodies and objects in adults with autism spectrum disorder. Journal of Autism and Developmental Disorders 44(9): 2277-2289.

Presentation (Computer Software) (2014) Available at: www. neurobs.com

Sachse M, Schlitt S, Hainz D, et al. (2013) Executive and visuomotor function in adolescents and adults with autism spectrum disorder. Journal of Autism and Developmental Disorders 43(5): 1222-1235.

Shepard RN and Metzler J (1971) Mental rotation of threedimensional objects. Science 171(3972): 701-703.

Silk TJ, Rinehart N, Bradshaw JL, et al. (2006) Visuospatial processing and the function of prefrontal-parietal networks in autism spectrum disorders: a functional MRI study. American Journal of Psychiatry 163(8): 1440-1443.
Soulières I, Zeffiro TA, Girard ML, et al. (2011) Enhanced mental image mapping in autism. Neuropsychologia 49(5): $848-857$.

Tapley SM and Bryden MP (1977) An investigation of sex differences in spatial ability: mental rotation of three-dimensional objects. Canadian Journal of Psychology 31(3): 122-130.

Valdez P, Reilly T and Waterhouse J (2008) Rhythms of mental performance. Mind, Brain, and Education 2: 7-16.

Voracek M and Dressler SG (2006) Lack of correlation between digit ratio (2D:4D) and Baron-Cohen's 'Reading the Mind in the Eyes' test, empathy, systemising, and autism-spectrum quotients in a general population sample. Journal of Clinical and Experimental Neuropsychology 41: 1481-1491.

Voyer D, Voyer S and Bryden MP (1995) Magnitude of sex differences in spatial abilities: a meta-analysis and consideration of critical variables. Psychological Bulletin 117(2): 250-270.

Werling DM and Geschwind DH (2013) Sex differences in autism spectrum disorders. Current Opinion in Neurology 26(2): 146-153.

Zapf AC, Glindemann LA, Vogeley K, et al. (2015) Sex differences in mental rotation and how they add to the understanding of autism. PLOS ONE 10(4): e0124628. 